\title{
GESTÃO DO NÍVEL DE DETALHAMENTO DA INFORMAÇÃO EM UM MODELO BIM: ANÁLISE DE UM ESTUDO CASO ${ }^{1}$
}

\section{LEVEL OF DEVELOPMENT MANAGEMENT IN A BIM MODEL: ANALYSIS OF A CASE STUDY}

\author{
Joyce Paula Martin Delatorre \\ Universidade de São Paulo (USP) \\ joyce.delatorre@usp.br \\ Eduardo Toledo Santos \\ Universidade de São Paulo (USP) \\ etoledo@usp.br
}

\begin{abstract}
Resumo
Uma das dificuldades do processo de implementação do BIM que está dificultando a completa adoção pelo mercado brasileiro é à definição e mensuração do nível de desenvolvimento dos modelos ao longo de cada etapa do processo de projeto. Através de revisão bibliográfica e da análise do processo de gestão das informações do modelo em um estudo de caso, foi verificado que a falta de uma definição clara dos usos que se almeja do modelo BIM e da definição do nível de detalhamento das informações do modelo pode gerar problemas de comunicação e o não atingimento dos usos e das expectativas dos envolvidos, demonstrando a importância da definição de um processo de gestão do nível de detalhamento da informação BIM. Como resultado, foi feita a proposição de um macro fluxo para a gestão do nível de desenvolvimento de modelos BIM. Acredita-se que os dados gerados a partir desta pesquisa, bem como a abordagem aplicada, possam contribuir para novas investigações com relação à proposição de um modelo para a gestão do nível de desenvolvimento do modelo BIM durante o processo de desenvolvimento de projeto e como orientação para o acompanhamento da evolução do modelo em projetos desenvolvidos em BIM.
\end{abstract}

Palavras-chave: Modelagem da Informação da Construção. BIM. Nível de desenvolvimento.

\begin{abstract}
The definition and measurement of the level of development of the BIM models throughout each phase of the design process is one of the difficulties for the full adoption of the BIM implementation process in the Brazilian market. A literature review and the analysis of the information management process in a case study revealed that the lack of a clear definition of the expected BIM model uses and the expected level of detail for the elements in the model could generate communication problems, lead to the non-achievement of the uses, and result in unfulfilled stakeholders' expectations. It also showed the importance of defining a development management process of the amount of information in the BIM model. As a result, a macro flow for the management of the BIM model level of development was proposed. It is believed that the data generated from this research, as well as the applied approach, will contribute to further investigation in relation to the proposition of a model for the management of the BIM model level of development during the project development process. It will also serve as a guide for monitoring the evolution of the model in a BIM developed project.
\end{abstract}

Keywords: Building Information Modeling. BIM. Level of development.

\footnotetext{
${ }^{1}$ DELATORRE, J.P.M.; SANTOS, E.T. Gestão do nível de detalhamento da informação em um modelo BIM: Análise de um estudo de caso. In: ENCONTRO BRASILEIRO DE TECNOLOGIA DE INFORMAÇÃO E COMUNICAÇÃO NA CONSTRUÇÃO, 7., 2015, Recife. Anais... Porto Alegre: ANTAC, 2015.
} 


\section{8}

\section{TIC2015}

\section{INTRODUÇÃO}

No mercado de construção civil, nos últimos anos, o termo BIM - "Building Information Modeling", ou Modelagem da Informação da Construção, deixou de ser um modismo com poucos pioneiros, para ser a peça central da tecnologia do mercado de Arquitetura, Engenharia e Construção (AEC), abordando aspectos de projeto, construção e operação de edifícios. A maioria das empresas líderes mundiais de arquitetura, engenharia e construção estão usando BIM em seus projetos. (EASTMAN et al., 2011).

Segundo Wilson e Heng (2011) BIM está mudando em um sentido mais amplo as práticas tradicionais de construção, em termos de pessoas, processos, trabalho, cultura, comunicação e modelos de negócio.

Reconhecendo a importância estratégica do uso de BIM e seguindo a tendência internacional, grandes contratantes brasileiros começam a exigir BIM em seus editais de concorrência. Pode-se citar, entre outras: Petrobras - Petróleo Brasileiro S/A; CDURP Companhia de Desenvolvimento Urbano da Região do Porto do Rio de Janeiro; INPI Instituto Nacional de Propriedade Industrial (CONSTRUÇÃO MERCADO, 2011); DNIT Departamento Nacional de Infraestrutura de Transportes (DINIZ, 2012) e CPTM Companhia Paulista de Trens Metropolitanos.

Embora pequenas e grandes empresas tenham iniciado o processo de implementação e uso do BIM, há diversas dificuldades no processo de implementação que estão dificultando a completa adoção do mercado brasileiro. Souza, Amorim e Lyrio (2009) identificaram alguns destes problemas imediatos na transição da tecnologia tradicional atual para novos sistemas de informação: alto custo dos softwares, resistência à mudança pela equipe e falta de integração entre projetistas, entre outros.

Usuários mais avançados reconhecem dificuldades de outras naturezas. Uma delas está relacionada à definição e mensuração do nível de desenvolvimento do modelo. Segundo o BIM Forum (2015), a precisão de um elemento do modelo pode ser interpretada de forma errada uma vez que um componente genérico, numa posição aproximada pode ter a mesma aparência de um elemento específico, posicionado precisamente. Desta forma, são necessárias outras considerações além da aparência para definir a precisão de um determinado elemento.

O objetivo deste artigo é analisar como é abordada esta gestão nos referenciais teóricos, verificar em um estudo de caso como foi feita a gestão desta informação e, a partir da análise dos resultados alcançados, propor um macro fluxo para a gestão do nível de desenvolvimento de modelos BIM.

\section{METODOLOGIA}

O método de pesquisa adotado para esta investigação consistiu na realização de revisão bibliográfica aprofundando conceitos teóricos sobre as fases de desenvolvimento de projetos no modelo tradicional e no processo BIM e na análise de um estudo de caso em empresa construtora que está envolvida em projetos que estão sendo desenvolvidos com o uso da tecnologia BIM. Com base na revisão da literatura e dos pontos de atenção identificadas no processo adotado no estudo de caso, foi feita a proposição de um macro fluxo para a gestão do nível de desenvolvimento de modelos BIM.

A adoção do estudo de caso como método de investigação se deu devido à pesquisa estar relacionada com a análise de uma situação, no caso, o nível de detalhamento das informações de um projeto desenvolvido a partir do uso da tecnologia BIM, dentro da realidade das empresas de construção civil. Segundo Martins (2008), o estudo de caso é uma investigação empírica que pesquisa fenômenos dentro de seu contexto real, no qual o 
pesquisador não tem controle sobre os eventos e variáveis. Com isso, procura-se apreender a totalidade de uma situação e, criativamente, descrever, compreender e interpretar a complexidade de um caso concreto. O Estudo de Caso possibilita a penetração na realidade que não é conseguida plenamente pela avaliação quantitativa, através de um estudo profundo e exaustivo de um objeto delimitado que é o problema de pesquisa.

Este estudo de caso foi realizado através de entrevista com a coordenadora do projeto por parte da construtora, da análise da documentação do projeto (manual do projeto BIM, contratos, relatórios de análise crítica do modelo), e da análise do modelo de arquitetura entregue em cada uma das fases do projeto, verificando o nível de detalhamento da informação nele contida.

Por fim, foi feita a análise dos dados obtidos e confronto com a literatura estudada para concluir como foi a gestão do nível de detalhamento da informação do modelo BIM no estudo de caso e quais foram os resultados desta gestão. A partir desta análise, foi feita a proposição de um macro fluxo para a gestão do nível de desenvolvimento de modelos BIM.

\section{AS ETAPAS DE DESENVOLVIMENTO DO PROJETO E O NíVEL DE DETALHAMENTO DA INFORMAÇÃO}

No Brasil, dentre as principais referências que detalham as fases de projeto estão:

- Manual de Contratação de Serviços de Arquitetura e Urbanismo, desenvolvido pela Associação Brasileira de Escritórios de Arquitetura (AsBEA). (AsBEA, 1992).

- Norma NBR 13531 - Elaboração de projetos de edificações - Atividades técnicas Procedimento, desenvolvida pela Associação Brasileira de Normas Técnicas - ABNT. (ABNT, 1995).

- Manual de escopo de serviços para a Coordenação de Projetos, desenvolvido com a coordenação geral do SECOVI/SP, com o apoio técnico da SINDINSTALAÇÃO, SINDUSCON e das entidades de classe AGESC, ABECE, ABRASIP, ABRAVA e AsBEA. (SECOVI/SP, 2012).

De acordo com Tzortzopoulos (1999), os principais objetivos propostos pelo Manual da AsBEA (1992) incluem a configuração do parâmetro base para a fixação de honorários profissionais; a normalização dos procedimentos para a elaboração coordenada do projeto de arquitetura de edificações; a discriminação dos serviços incluídos e excluídos nos contratos que tenham por objetivo o projeto de arquitetura de edificações; a definição e caracterização dos principais elementos técnicos relacionados ao projeto, em especial as fases e etapas que o compõem, as informações necessárias ao seu desenvolvimento, e os produtos finais e serviços que o caracterizam. As cinco primeiras etapas se referem à sequência de desenvolvimento de projetos: levantamento de dados; estudo preliminar, anteprojeto, projeto legal e projeto executivo. Este último pode ser subdividido em até quatro subfases: pré-executivo, projeto básico, projeto de execução, e detalhes de execução. As demais etapas previstas podem ser desenvolvidas ao longo de todo o processo e incluem a coordenação e o gerenciamento dos projetos, assistência à execução e serviços adicionais.

A Norma NBR 13531 (ABNT, 1995) fixa as condições exigíveis para a elaboração de projetos de edificações aplicáveis a todas as classes tipológicas das edificações, tais como: habitacional, educacional, cultural, religiosa, comercial, industrial, administrativa, esportiva, de saúde, de lazer, de comunicação, de transporte, de abastecimento e de segurança. De acordo com a Norma, as etapas de projeto devem possibilitar a subsequente definição e articulação das etapas das demais atividades técnicas que compõem o projeto da edificação e são subdivididas em: levantamento de dados (LV), programa de necessidades (PN), estudo de viabilidade (EV), estudo preliminar (EP), anteprojeto (AP) ou pré-execução (PR), 
projeto legal $(\mathrm{PL})$, projeto básico $(\mathrm{PB})$ que é considerado opcional, projeto de execução (PE). Para cada uma das etapas, a Norma orienta com as informações de referência a utilizar, informações técnicas a produzir e documentos técnicos a apresentar. Segundo Tzortzopoulos (1999) a norma apresenta lacunas e indefinições, apresentando de forma genérica a subdivisão dos processos de projeto e do empreendimento.

O manual de escopo de serviços para a Coordenação de Projetos (SECOVI/SP, 2012) define e detalha, de forma abrangente, o fluxo de atividades exigido para a coordenação de projetos imobiliários. O processo de projeto é dividido em seis fases principais: Fase A Concepção do produto; Fase B - Definição do Produto; Fase C - Identificação e Solução de Interfaces de Projeto; Fase D - Detalhamento de Projetos; Fase E - Pós Entrega de Projetos e Fase F - Pós-Entrega da Obra. Para cada serviço previsto são definidos os dados necessários, a descrição das atividades e os produtos gerados em cada fase.

Quadro 1 - Resumo comparativo das propostas de etapas de projeto da AsBEA, NBR 12331 e SECOVI

\begin{tabular}{|c|c|c|c|}
\hline Referência & AsBEA & NBR 13531 & Secovi \\
\hline Ano & 1992 & 1995 & 2012 \\
\hline Geral & $\begin{array}{l}\text { Parâmentro base para a fixação } \\
\text { de honorários profissionais; } \\
\text { A normalização dos } \\
\text { procedimentos para a elaboração } \\
\text { coordenada do projeto de } \\
\text { arquitetura de edificações; } \\
\text { A discriminação dos serviços } \\
\text { incluídos e excluídos nos } \\
\text { contratos que tenham por objetivo } \\
\text { o projeto de arquitetura de } \\
\text { edificações; } \\
\text { A definição e caracterização dos } \\
\text { principais elementos técnicos } \\
\text { relacionados ao projeto, em } \\
\text { especial as fases e etapas que o } \\
\text { compõem, as informações } \\
\text { necessárias ao seu } \\
\text { desenvolvimento, e os produtos } \\
\text { finais e serviços que o } \\
\text { caracterizam. }\end{array}$ & $\begin{array}{l}\text { Fixa as condições } \\
\text { exigíveis para a } \\
\text { elaboração de projetos } \\
\text { de edificações } \\
\text { aplicáveis a todas as } \\
\text { classes tipológicas das } \\
\text { edificações; } \\
\text { Orienta com as } \\
\text { informações de } \\
\text { referência a utilizar, } \\
\text { informações técnicas a } \\
\text { produzir e documentos } \\
\text { técnicos a apresentar. }\end{array}$ & $\begin{array}{l}\text { Define e detalha de forma } \\
\text { abrangente o fluxo de } \\
\text { atividades exigido para a } \\
\text { coordenação de projetos } \\
\text { da indústria imobiliária. } \\
\\
\text { Para cada serviço } \\
\text { previsto são definidos os } \\
\text { dados necessários a } \\
\text { descrição das atividades } \\
\text { e os produtos gerados em } \\
\text { cada fase. }\end{array}$ \\
\hline $\begin{array}{l}\text { Etapas } \\
\text { Propostas }\end{array}$ & $\begin{array}{l}\text { - Levantamento de dados; } \\
\text { - Estudo preliminar; } \\
\text { - Anteprojeto; } \\
\text { - Projeto Legal; } \\
\text { - Projeto Executivo (pode ser } \\
\text { subdividido em pré-executivo; } \\
\text { projeto básico; projeto de } \\
\text { execução e detalhes de } \\
\text { execução) }\end{array}$ & $\begin{array}{l}\text { - Levantamento de } \\
\text { dados (LV); } \\
\text { - Programa de } \\
\text { necessidades (PN); } \\
\text { - Estudo de } \\
\text { viabilidade (EV); } \\
\text { - Anteprojeto (AP) ou } \\
\text { pré-execução (PR); } \\
\text { - Projeto legal (PL); } \\
\text { - Projeto básico (PB) } \\
\text { (opcional); } \\
\text { - Projeto de execução } \\
\text { (PE). }\end{array}$ & $\begin{array}{l}\text { - Fase A - Concepção do } \\
\text { produto; } \\
\text { - Fase B - Definição do } \\
\text { produto; } \\
\text { - Fase C - Identificação e } \\
\text { Solução de Interfaces } \\
\text { de Projeto; } \\
\text { - Fase D - Detalhamento } \\
\text { de projetos; } \\
\text { - Fase E - Pós-Entrega } \\
\text { de projetos; } \\
\text { - Fase F - Pós-Entrega } \\
\text { da obra. }\end{array}$ \\
\hline
\end{tabular}


Com base na análise do resumo comparativo entre as fases de projeto propostas pela AsBEA, ABNT e Secovi (Quadro 1), as referências encontradas abordam apenas as diretrizes sobre as informações de entrada, as atividades a serem desenvolvidas e os produtos a serem entregues. Não foi possível constatar uma uniformidade e detalhamento do nível das informações de projeto necessárias por fase. A estrutura de fases e o nível de detalhamento são apresentados de maneira geral e de forma distinta e os referenciais indicam ainda que as diretrizes apresentadas podem ser adaptadas, considerando as particularidades de cada empreendimento e as necessidades e expectativas de cada contratante. Portanto, foi possível constatar que as referências brasileiras que detalham as fases de projeto não estão adequadas para trocas de informações em BIM, uma vez que foram desenvolvidas com base apenas no nível de informação contida em documentos 2D.

\section{O NÍVEL DE DETALHAMENTO DA INFORMAÇÃO DO MODELO BIM E OS REFERENCIAIS TEÓRICOS}

Diversas referências teóricas tratam do nível de detalhamento da informação dentro do contexto BIM.

Segundo o Computer Integrated Construction Research Group (2012), o nível de desenvolvimento (LD) proposto pela AIA (American Institute of Architects) é o mais amplamente utilizado na indústria nos EUA. Este descreve as dimensões quantitativa e qualitativamente, além de outros dados mínimos que devem ser incluídos no modelo para apoiar um uso autorizado associado a esse nível de detalhe. A estrutura do nível de desenvolvimento (LOD) dá subsídio aos participantes para o entendimento da evolução de um elemento da ideia conceitual até a definição precisa. Esta estrutura informa aos participantes em que nível de desenvolvimento uma informação deve estar em uma determinada fase do projeto, o que ajuda a evitar que algum participante utilize o modelo de forma não pretendida pelo seu autor ou infira maior precisão do que a real, permitindo que os participantes se comuniquem de maneira eficiente.

Figura 1 - Tabela para definição do nível de detalhamento de cada elemento do modelo: AIA Document G202TM-2013 - Project Building Information Modeling Protocol Form

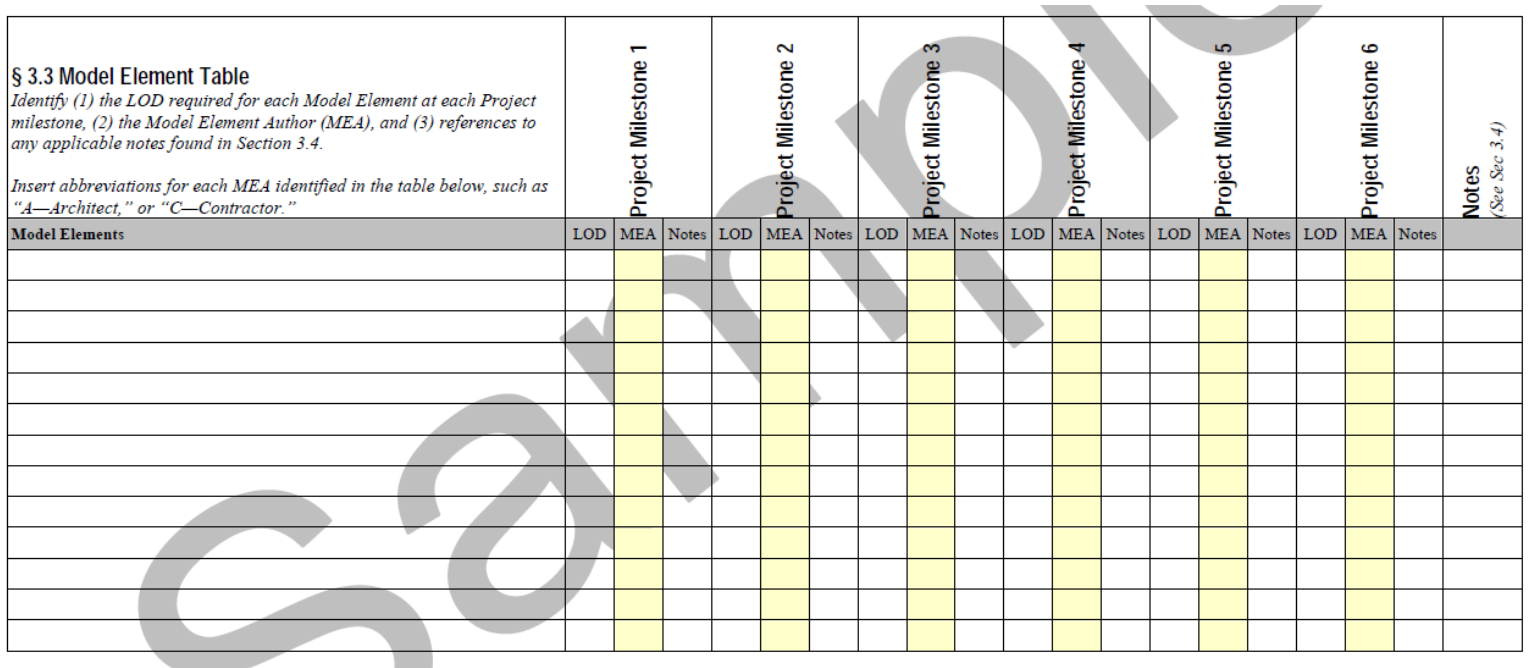

Fonte: AIA, 2013

Para facilitar a comunicação entre os participantes do projeto e a correta interpretação do quadro de LOD proposto pela AIA (Figura 1), o BIM Forum (2015) criou um padrão para a indústria descrevendo o estado de desenvolvimento de vários elementos construtivos, 
incluindo imagens representativas, com o objetivo de garantir a consistência na comunicação e execução, facilitar a definição detalhada dos marcos BIM e resultados, mas não tem como objetivo prescrever os níveis de desenvolvimento que devem ser alcançados em cada etapa do projeto (Figura 2).

Figura 2 - Exemplo de ilustração no documento do BIM Forum para auxiliar na compreensão do nível de detalhamento proposto

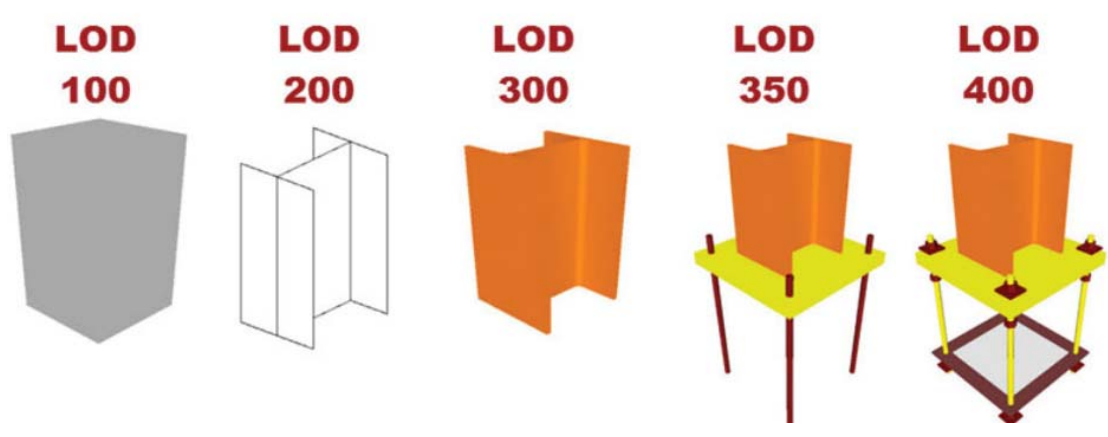

Fonte: BIM Forum (2015)

Os objetivos gerais do documento do BIM Forum (2015) são:

- ajudar as equipes, incluindo proprietários, a especificar entregas BIM e definir uma imagem clara do que será incluído no modelo;

- ajudar os gerentes de projeto a explicar para as suas equipes a informação e detalhe que deve ser fornecida em várias etapas do processo de desenvolvimento de projeto;

- permitir que os usuários a jusante possam contar com informações específicas de modelos que foram recebidos de outros; proporcionar um padrão que pode ser referenciado em contratos e em planos de execução BIM.

Segundo o BIM Forum (2015), não existe uma correspondência rigorosa entre o LOD e as fases de projeto, pois o desenvolvimento de cada um dos sistemas ocorre em velocidades diferentes. O modelo entregue em uma determinada etapa do projeto pode conter elementos modelados em vários níveis de desenvolvimento. O mesmo documento traz um importante esclarecimento sobre a diferença entre Nível de Detalhe (Level of Detail) e Nível de Desenvolvimento (Level of Development), conceitos comumente confundidos, já que têm a mesma sigla (LoD): o primeiro conceito se refere à quanto detalhe (geométrico e de informação) foi inserido no elemento (entrada de informação). O segundo, e mais importante, diz respeito ao grau de detalhe de informação confiável presente no elemento (informação confiável que pode ser extraída do elemento). Como exemplo, um projetista pode inserir, no modelo BIM da fase de anteprojeto, um componente representando um aquecedor de água a gás, obtido do site de um fabricante. Isso não quer dizer que a potência, capacidade, posição das entradas/saídas de água e gás, dimensões, etc. já estejam definidas e especificadas pelo projetista (que o fará num ponto mais tarde no ciclo de projeto), apesar de todas essas informações provavelmente já constarem no elemento inserido no modelo. Nessa etapa, apenas se poderia inferir a existência e posição aproximada de um aquecedor a gás no projeto, a partir do componente BIM inserido.

O Computer Integrated Construction Research Group - CIC (2011) destaca a importância para os membros da equipe, em especial, o autor e receptor, do entendimento claro do conteúdo da informação em um processo de troca de informações e propõe a definição de três níveis de detalhamento: A - Tamanho e localização precisos com inclusão de parâmetros de objetos e de materiais; B - Tamanho e localizações gerais, inclusão de 
parâmetros; C - Tamanho e localizações esquemáticas. Para cada elemento do modelo, deve-se atribuir o responsável pela criação da informação requerida (Figura 3).

Figura 3 - Tabela para definição do nível de detalhamento da informação proposto pelo $\mathrm{CIC}$

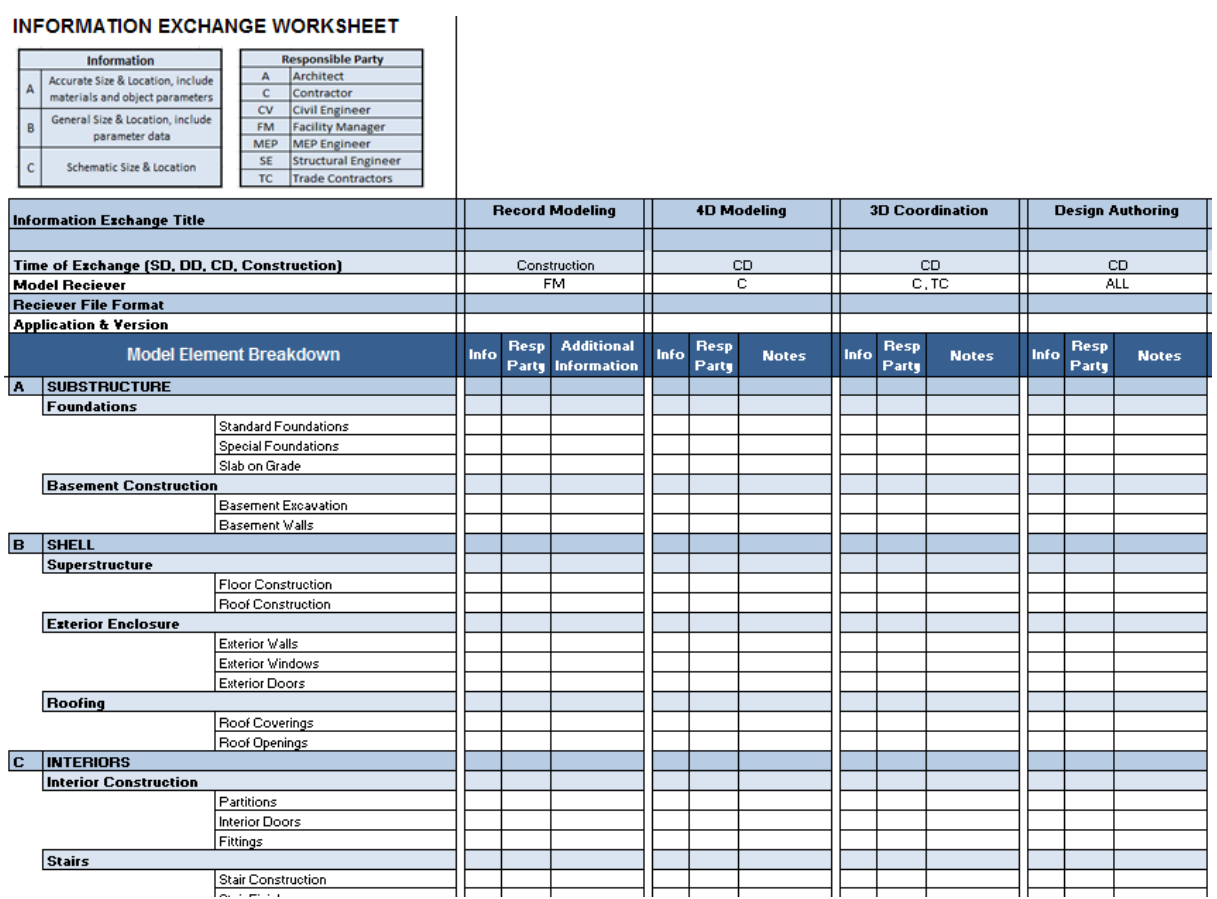

Fonte: Computer Integrated Construction Research Group, 2011.

\section{CASO}

O caso apresentado neste artigo foi desenvolvido a partir da análise da documentação de um projeto desenvolvido em BIM de uma construtora. Trata-se de um edifício de uso misto em São Paulo, com um hotel com mais $40 \mathrm{mil} \mathrm{m}^{2}$ de área construída e unidades corporativas com aproximadamente $17 \mathrm{mil} \mathrm{m}^{2}$.

Neste projeto, a bandeira hoteleira exigiu a participação de projetistas homologados por ela, o que levou à contratação de projetistas internacionais para o desenvolvimento do projeto e projetistas locais para acompanhar o desenvolvimento, como consultores, desde o início do projeto e assumir o projeto executivo.

Como o cliente decidiu pela adoção do processo BIM no projeto, contratou uma consultoria BIM para fazer a gestão do mesmo.

Com base na análise dos documentos utilizados durante a gestão do projeto BIM e de entrevista com a coordenadora do projeto, pode-se constatar que, embora citado na solicitação da proposta, o nível de detalhamento do modelo não foi claramente alinhado entre todos os envolvidos, gerando problemas de comunicação e de troca de informação entre os agentes envolvidos. 
Quadro 2 - Resumo dos requisitos BIM citados nos documentos de contratação do projeto

Requisitos BIM citados nos documentos contratuais do projeto

Informações relacionadas aos requisitos BIM contidas na requisição de proposta do cliente para contratação da construtora

- declaração de que uma empresa especializada para o gerenciamento do BIM e de todos os requisitos envolvidos no processo estará envolvida no projeto;

- solicitação que a proponente disponibilize uma equipe de BIM com profissionais especialistas;

- solicitação que o modelo seja desenvolvido de acordo com as etapas de entrega do projeto;

- informação de que o nível de detalhamento (LOD) para as diferentes fases e padrões dos modelos e as informações serão fornecidas em conjunto com um manual de BIM;

- solicitação que a proponente deve assegurar que o banco de dados BIM esteja integrado e coordenado;

- solicitação que a proponente garanta que os modelos BIM estejam compatibilizados com os desenhos de outras disciplinas contratadas.

Informações contidas no manual do projeto citado na requisição de proposta

- não foi localizada informação sobre o detalhamento do LOD citado na requisição de projeto

Fonte: Autor

Além disso, foi constatada uma lacuna entre as informações esperadas e recebidas entre a Incorporadora, Construtora e Projetista, potencializada pela falta de determinação sobre a matriz de responsabilidade sobre as informações do modelo. A coordenadora do projeto relatou que houve algumas iniciativas por parte da construtora em definir o nível de detalhamento proposto entre os participantes, porém sem formalização por parte do contratante. O cliente, neste caso, se baseou na proposta da AIA para definição do LOD, porém não seguiu o principio de definição por tipologia de elemento. Como uma das consequências, a coordenadora exemplificou que os usos que esperava do modelo para a construção não puderam ser concretizados pela ausência de detalhamento necessário no modelo.

A análise da evolução do nível de detalhamento da informação conforme a evolução das fases do projeto demonstrou que, embora o número total de elementos tenha aumentado de uma fase para a outra, o nível de informação contida nos elementos não evoluiu significativamente de uma fase para outra.

\section{RESULTADO}

Ao se analisar o estudo de caso com base no macro fluxo proposto, pode-se constatar que não foi dada a importância necessária à gestão do nível de detalhamento do modelo no projeto, gerando retrabalho, problemas de comunicação e não atingimento dos objetivos esperados pelos participantes para o modelo.

Embora o contratante deste projeto tenha optado pelo uso do BIM e pela contratação de consultoria especializada para auxiliar no processo de gestão BIM, nenhum documento formalizou os usos e objetivos pretendidos do modelo por fase.

O nível de detalhamento esperado do modelo e a matriz de responsabilidades também não foram acordados entre os participantes, gerando expectativas entre os envolvidos que não foram atingidas, uma vez que o processo não foi desenhado para o seu atingimento. 
Com base no conteúdo dos relatórios de análise crítica do modelo, pode-se verificar que estes apresentaram uma avaliação superficial, sem a utilização de métricas de qualidade que embasem a análise. Foi demonstrado que, embora o nível de detalhamento dos elementos do modelo não apresentasse evolução significativa de uma fase para a outra, os envolvidos no projeto não tinham a visão clara deste fato.

Por fim, pôde-se inferir que, sem a constatação clara da baixa evolução dos elementos do modelo e na ausência de definição clara de métricas, a retroalimentação do processo e o direcionamento para que o projeto atinja o resultado esperado foram dificultados.

Com base no resultado desta análise, propõe-se o seguinte macro fluxo com as principais etapas que devem ser consideradas para o processo de gestão do nível de desenvolvimento do modelo.

Figura 4 - Macro-fluxo proposto para a gestão do nível de desenvolvimento dos elementos do modelo
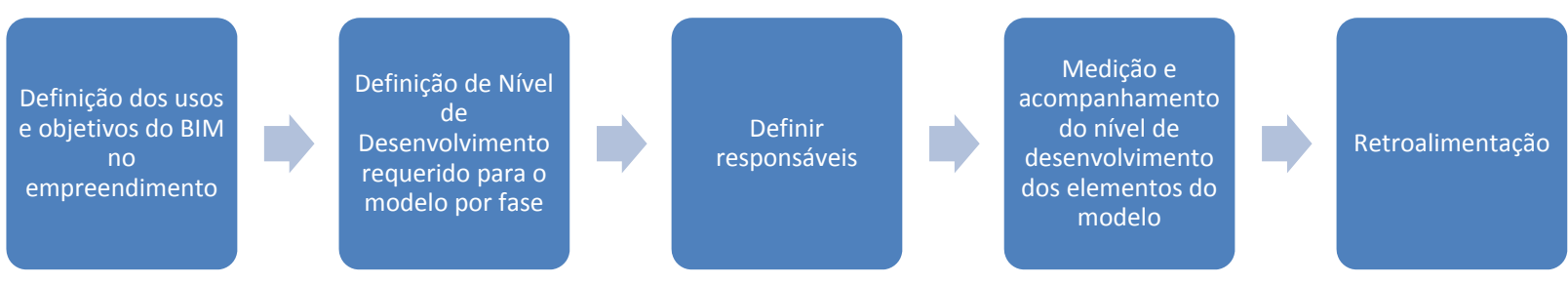

Fonte: Autor

\section{CONCLUSÃO}

Uma das dificuldades relacionadas ao processo de implantação de BIM em um projeto é a gestão do nível de desenvolvimento no que se refere à definição e mensuração da evolução das informações contidas no modelo. Com base na revisão bibliográfica, constatou-se a necessidade de que esta definição seja requerida tanto por elemento construtivo quanto por etapa do projeto, de maneira a subsidiar usos pré-definidos pelos agentes envolvidos.

Embora grande parte das referências bibliográficas aponte a necessidade da definição dos usos que se almeja do modelo BIM e da definição do nível de detalhamento das informações do modelo por etapa de projeto de acordo com os usos que são esperados pelos envolvidos no processo, na prática é possível constatar que existem empresas que aplicam o processo BIM sem um alinhamento claro destas informações entre as partes.

É possível inferir pela análise do estudo de caso que a falta deste alinhamento pode gerar problemas de comunicação e o não atingimento dos usos e das expectativas dos envolvidos, demonstrando a importância da definição de um processo de gestão do nível de detalhamento da informação BIM.

Pela revisão da bibliografia, verificou-se que, dentre os principais pontos a serem incluídos dentro do processo de gestão BIM, estão: definição dos usos e objetivos do modelo BIM; definição do nível de desenvolvimento requerido por elemento e por fase; definição dos responsáveis pela inserção das informações; utilização de uma metodologia para acompanhamento e medição do nível de desenvolvimento, e a retroalimentação do processo.

Acredita-se que o macro fluxo gerado a partir da revisão bibliográfica e da análise do estudo de caso possa ser aplicado em pesquisas futuras que investiguem a gestão do nível de desenvolvimento de modelos BIM e que os dados gerados possam contribuir para novas 
investigações com relação à proposição de um modelo para a gestão do nível de desenvolvimento do modelo BIM durante o processo de desenvolvimento de projeto.

\section{AGRADECIMENTOS}

O segundo autor agradece à FAPESP - Fundação de Amparo à Pesquisa do Estado de São Paulo e ao CNPq - Conselho Nacional de Desenvolvimento Científico e Tecnológico pelo apoio.

\section{REFERÊNCIAS}

AMERICAN INSTITUTE OF ARCHITECTS - AIA. AIA Document G202TM-2013 - Project Building Information Modeling Protocol Form. 2013. 5 p.

ASSOCIAÇÃO BRASILEIRA DE ESCRITÓRIOS DE ARQUITETURA - ASBEA. Manual de

Contratação de Serviços de Arquitetura e Urbanismo. São Paulo: Ed. PINI, 1992. 107 p.

ASSOCIAÇÃO BRASILEIRA DE NORMAS TÉCNICAS - ABNT. NBR 13.531. Elaboração de

Projetos de Edificações: Atividades técnicas. Rio de Janeiro, 1995. 10 p.

BIMFORUM. Level of Development Specification: For Building Information Models. v.2015-Draft, [S.I], 166 p. Disponível em:<http://bimforum.org/wp-content/uploads/2015/04/Files.zip>. Acesso em: 09 jun 2015.

Como anda o BIM nas incorporadoras. Construção Mercado, [S.I], ed. 143, jun. 2013. Disponível em: http://construcaomercado.kubbix.com/negocios-incorporacao-construcao/143/artigo2906921.aspx. Acesso em: 02. out. 2013.

COMPUTER INTEGRATED CONSTRUCTION RESEARCH GROUP - CIC. BIM Project Execution Planning Guide.v 2.1, The Pennsylvania State University, 2011, 125 p.

DINIZ, A.. Dnit exigirá nas licitações projetos entregues em formato BIM. O Empreiteiro, [S.I], ed. 513, 2012. Disponível em:< http://www.oempreiteiro.com.br/Publicacoes/6763/

Dnit_exigira_nas_licitacoes_projetos_entregues_em_formato_BIM.aspx >. Acesso em: 7 ago. 2015.

EASTMAN, C. M. et al. BIM Handbook: A Guide to Building Information Modeling for Owners, Managers, Designers, Engineers and Contractors, 2nd Edition. Hoboken: Wiley, 2011, 648 p.

MARTINS, G. A. Estudo de caso: uma estratégia de pesquisa. 2 ed. São Paulo: Atlas, 2008, 120 p.

SECOVI/SP (Sindicato das Empresas de Compra, Venda, Locação e Administração de Imóveis Residenciais e Comerciais de São Paulo). Manual de escopo de serviços para a Coordenação de Projetos: Indústria Imobiliária. São Paulo, 2012. 105 p.

SOUZA, L. L. A, AMORIM, S. R. L, LYRIO, A. M. Impactos do uso do BIM em escritórios de arquitetura: oportunidades no Mercado Imobiliário. Gestão \& Tecnologia de Projetos. v.4, n. 2, pag. 26-56, nov. 2009.

TZORTZOPOULOS, P. Contribuições para o desenvolvimento de um modelo de processo de projeto. 1999. 163 f. Tese (Dissertação de Mestrado) - Universidade Federal do Rio Grande do Sul, Porto Alegre. 1999.

LU, W.W. S. ; LI, H. Building information modeling and changing construction practices. Automation in Construction. v.20, n.2, pag. 99-100, 2011. 